\title{
Desafíos Interdisciplinarios en la Formación Universitaria: una Contribución desde la Facultad de Diseño de la Universidad del Desarrollo
}

\author{
Paulina del Pilar Contreras', Paula Rebeca Broitman²
}

\begin{abstract}
The ability to work in an interdisciplinary manner in order to respond to the creative challenges imposed by a complex and changing marketplace is an absolute necessity for professionals in modern day companies (CINDA, 2000), (OECD, 2005) where a product cannot be created by an individual alone. (Mamykina, Candy \& Edmonds, 2002). It generates an overlap of responsibilities between different professional disciplines and creates an interdependence between them in order to design and the produce innovative goods. The first part of this paper recognizes the necessity of incorporating creative and interdisciplinary abilities at all levels within a business and how this clearly extends to university programs. (Vanhamaa, 2006), (Boni, Weingart \& Evenson, 2012), (Itkonen, Ekman, \& Kojo, 2009). The second part presents how Universidad del Desarrollo responds to these challenges through the creation of the Institute for Interdisciplinary Innovation (Instituto de Innovación Interdisciplinaria ICUBO), which is born from the Departments of Design, Engineering and Economics and Business.

La capacidad de trabajo interdisciplinario para responder a los desafíos creativos que impone un mercado complejo y cambiante, es exigencia para los profesionales de la empresa actual (CINDA, 2000), (OECD, 2005), donde un producto no puede ser creado por un individuo (Mamykina, Candy \& Edmonds, 2002). Generando un traslape de responsabilidades entre profesionales de disciplinas distintas, e interdependencia para el diseño y producción de bienes innovadores. La primera parte de este paper reconoce la necesidad de incorporar capacidades creativas e interdisciplinarias a todo nivel de empresas y por ende a las mallas de estudios universitarios. (Vanhamaa, 2006), (Boni, Weingart \& Evenson, 20l2), (Itkonen, Ekman, \& Kojo, 2009). La segunda parte expone como la Universidad del Desarrollo responde a estos desafíos a través de la creación del Instituto de Innovación Interdisciplinaria ICUBO, que nace a partir de las Facultades de Diseño, Ingeniería y Economía y Negocios.
\end{abstract}

Keywords: interdisciplinary work; creative process; troubleshooting; design; innovation; trabajo interdisciplinario; proceso creativo; resolución de problemas; diseño; innovación.

Facultad de Diseño. Universidad del Desarrollo. Ainavillo 456, Concepción, Chile. 407000I.

Phone: +56 4l 22686 I0. email: 'paulinacontreras@udd.cl, ²pbroitman@udd.cl.

ISSN: 07 I8-2724. (http://www.jotmi.org)

Journal of Technology Management \& Innovation (C) Universidad Alberto Hurtado, Facultad de Economía y Negocios. 


\section{La producción centrada en el cliente}

La creciente aceptación de las reglas de libre mercado, ha ampliado el mercado global fomentando la competencia, la eficiencia y la creación de superávit entre las economías emergentes. Hace cuatro décadas, la demanda mundial de bienes era superior a la oferta (Parente, 2006) y la principal preocupación de las empresas era producir de manera rápida, eficiente y económica. El problema entonces no era vender sino producir.

Los actores más importantes del engranaje productivo eran, junto a los factores tradicionales (capital, recursos humanos, y tierra), eran los administradores (managers) que planeaban la asignación de recursos existentes (Albornoz, 20l I). A medida que el sistema capitalista continuó su expansión, se crearon economías de escalas y crecientemente más recursos son liberados para invertir en nuevos negocios y conocimientos (Perez, 2008). La acumulación de capital aumentó y la producción superó la demanda de los bienes más básicos, transformando el ambiente empresarial desde una perspectiva de producto a una perspectiva orientada al cliente, lo que implica innovación permanente para coincidir con lo que el cliente quiere y hacer posible la supervivencia del negocio. A medida que más empresas se enfocaban en segmentos específicos de mercado, la necesidad de una renovación permanente de sus productos y servicios convertía la creatividad de sus gerentes en una necesidad.

Las empresas establecidas deben hacer frente a un mercado extremadamente flexible que obliga a cambiar permanentemente sus estrategias de negocio. Esta característica siempre cambiante no sólo afecta a los propietarios de empresas ya establecidas, sino también a emprendedores que ambicionan entrar en el mercado.

Los empresarios de éxito deben ser creativos y aprender a un ritmo diferente, de lo contrario, no pueden sobrevivir. En este sentido, aprender más rápido que sus competidores se convirtió en la única ventaja competitiva sostenible en un entorno de rápido cambio y de innovación (De Geus, 1988). El mundo pasó de production driven a customer driven. Las empresas no sólo están enfocadas en producir con altos estándares de calidad, sino además, se enfrentan al permanente desafío de satisfacer clientes cada vez más variados y cambiantes. Los servicios cobran gran importancia, requiriendo de diversos tipos de conocimiento en la creación de procedimientos y métodos de prestación, provocando nuevos modelos de comportamiento en las organizaciones y personas, como es el concepto de service science, desarrollado por IBM, el cual busca desarrollar una visión sistémica en la empresa (Spohrer \& Maglio, 2008). La compleja y creciente oferta de bienes con atributos cada vez más diferenciados e innovadores desafía las capacidades creativas de la empresa.
Provocando que la producción, la usabilidad, y la creatividad estén más vigentes que nunca.

Esta intersección entre producción, usabilidad y creatividad, pone a las disciplinas diferentes, como son por ejemplo: diseño, ingeniería, economía y negocios, en un traslape de responsabilidades, generando una interdependencia para el diseño y producción de productos y servicios. Sin embargo ¿existen parámetros para educar este tipo de trabajo, ahora sistemático e interdisciplinario?

Uno de los elementos centrales que requiere la unión de las disciplinas de diseño, ingeniería, economía y negocios, es la capacidad de desarrollar un proceso creativo interdisciplinario para enfrentar los desafíos creativos de la empresa; creación de nuevos sistemas productivos, optimización de los mismos, desarrollo de productos y servicios e interfaces complejos de sofisticado diseño, logística y comercialización, entre otros. Todas estas actividades de la empresa, requieren trabajo conjunto para desarrollar soluciones que no pueden ser abordadas por un individuo solo. Convirtiendo la capacidad de trabajo interdisciplinario una exigencia para el profesional actual.

El proceso creativo siempre ha sido relevante para la disciplina del diseño, siendo una de sus competencias y metodologías más importantes (MacDonald, 20II). Pero, actualmente, el proceso creativo en equipos disciplinarios de diseño no es suficiente. El diseño responde a necesidades interdisciplinarias. Las oficinas de diseño se mueven de un campo a otro abarcando cada vez más áreas económicas en las que anteriormente el diseño no era requerido, como son los sectores de minería, agroindustria, forestal, entre otros ámbitos hasta ahora, más cercanos a la ingeniería y/o economía y negocios. Esto ha implicado que el diseño se tenga que adaptar a procesos creativos interdisciplinarios con disciplinas muy distintas, involucrando versatilidad y flexibilidad en la comunicación con los profesionales de otras áreas. Lo cual hace necesario incorporar el proceso creativo interdisciplinar en las mallas de estudio, a fin de lograr futuras optimizaciones del mismo en la empresa.

\section{El proceso creativo interdisciplinario}

La Creatividad se expresa en todos los ámbitos del quehacer humano, desde lo cotidiano en la forma de resolver los distintos desafíos que se nos presentan en la vida, hasta ámbitos técnicos y científicos, como herramienta para el desarrollo del ejercicio profesional.

Ken Robinson (2006), señala que la creatividad es tan importante en la educación como la alfabetización, por lo que de acuerdo con este autor deben tratarse con la misma importancia. Este postulado expone la necesidad de que la edu- 
cación forme personas autónomas, dinámicas, creativas, capaces de generar soluciones y respuestas frente a un mundo cada vez más complejo.

Respecto al proceso creativo, existen diferentes enfoques que lo definen. Wallas (1926), identifica 4 fases de éste proceso: Preparación, Incubación, lluminación, y Verificación. Su propuesta ha servido como base para estudios posteriores, pero como afirma Csikszentmihalyi (1998), ha sido una forma demasiado simplificada para definir un proceso tan complejo. Muchos autores han redefinido la postura de Wallas, como son Osborn (1960) y Patrick (1965). Según Torrance (1988), se puede detectar que el proceso propuesto por Wallas es la base de todas las teorías, métodos o disciplinas metodológicas en la formación existente en todo el mundo. Como expone Obradors (2007) en su libro "Creatividad y Generación de Ideas"; dentro de las definiciones de creatividad que tienen en cuenta el proceso, están las que consideran el inconsciente como principal artífice de la creatividad, Freud (198I), Wallas (1926), Poincaré (1913), Koestler (1964); las que defienden la existencia de un inconsciente colectivo, Jung (1994); las que defienden la intervención del preconsciente en el proceso creativo, Kubie (1958) y Kris (1952); Las que definen la existencia de una fase imprescindible de incubación inconsciente en el proceso creativo, Wallas (1926), Poincaré (1913), Koestler (1964); y las que no comparten esta consideración, definiendo que la fase de incubación inconsciente no parece ser necesaria en el proceso de generación de ideas, sino que el proceso creativo implica una búsqueda activa, un planteamiento de problemas y el objetivo claro de llenar agujeros de conocimiento que requieren una atención consciente, Gruber (1984), Gardner (1995), Perkins (198I), Csikszentmihalyi (1988), Weisberg (1987), Boden 1994, Sternberg (1997), Romo (1997).

La creatividad es una de las habilidades que debieran estar presentes en la vida profesional, para llegar a conclusiones nuevas y resolver problemas de manera útil y original (Carevic, 2006). Kauffman y Beguetto (2009) reconocen 4 niveles de creatividad, entre ellas la creatividad "Pro-c": creatividad profesional enfocada a una respuesta útil o formal. Es este nivel de creatividad individual es el que debe potenciarse a nivel de equipos interdisciplinarios profesionales.

En el pasado la investigación sobre creatividad se centró en los procesos cognitivos individuales, pero investigaciones recientes demuestran un cuadro más complicado de la creatividad. Se destaca la importancia de la interacción social, el seguimiento y la colaboración en el trabajo creativo. (Mamykina, et al., 2002). "La creatividad implica muchos factores sicológicos, seguirá creciendo como un campo interdisciplinario" (Xu, et al., 2005).

Hay una serie de diferencias importantes entre las fuerzas impulsoras de la creatividad individual y colaborativa. La importancia de analizar la creatividad en este sentido más holístico, es evidente si se considera que las actividades más creativas de la industria requieren de la participación de equipos interdisciplinarios que trabajan en conjunto para desarrollar un producto que no puede ser creado por un individuo solo. (Mamykina et al., 2002).

"Engineering Design Thinking, Teaching, and Learning" (Dym, Acogino, Eris, Frey \& Leifer, 2005) revisa la historia del rol del diseño en el currículum de ingeniería. De aquí se extrae que ambas disciplinas (diseño e ingeniería) diseñan, pero su concepción, métodos y aportes de diseño son diferentes. $Y$ ambas disciplinas requieren de proceso creativo para resolver problemas.

\section{La interdisciplinearidad}

Existe preocupación sobre la relevancia del trabajo interdisciplinario desde la formación universitaria en todas las carreras. En el área del diseño, el investigador británico Blair (20II) destaca la necesidad de integrar el diseño a la interdisciplina en la empresa.Vanhamaa (2006) plantea que la formación en industria es una tradición en los estudios de ingeniería, pero eso no suele ser el caso en el campo del diseño. Hoy en día, la creciente competencia en los negocios exige que diseño y tecnología deban estar perfectamente integrados. Los diseñadores deben ser empleados cada vez más en la industria, además de realizar consultorías empresariales. Otro ejemplo es la Campaña de Formación Murjottelu lanzada por Helsinki University of Technology, que planteó el proyecto de armar parejas conformadas por un estudiante de diseño industrial y un estudiante de ingeniería y ofrecer la pareja a las empresas industriales. A medida que la Campaña se ha ampliado, ha demostrado resultados donde la interdisciplinariedad, en general, ha crecido a un papel más importante. La pareja de estudiantes es más creativa, eficaz y autónoma que los alumnos singulares en el formato tradicional de formación. Es evidente que hay muchas posibilidades para incrementar productividad, creatividad e interacción entre negocios y academia introduciendo una programa de entrenamiento mejor organizado para estudiantes de diseño y de ingeniería (Itkonen et al., 2009). Esto propone serios desafíos para las industrias y para los propios diseñadores.

La bibliografía estudiada enfatiza el enfoque interdisciplinario que debe abordar el diseño, ya que su valor se potencia y proyecta en el trabajo en equipos interdisciplina. En el área de Ingeniería, destaca el reporte de la Escuela de Ingeniería de la Universidad de Michigan (Duderstadt, 2008), que plantea lo siguiente: Las nuevas tecnologías y los problemas complejos de los megaproyectos que surgen en la sociedad contemporánea requieren equipos de ingenieros altamente interdisciplinarios, con habilidades mucho más amplias que

ISSN: 07I 8-2724. (http://www.jotmi.org)

Journal of Technology Management \& Innovation @ Universidad Alberto Hurtado, Facultad de Economía y Negocios. 
simplemente el dominio de las disciplinas científicas y tecnológicas, requiriendo habilidades profesionales, tales como la innovación y el espíritu empresarial. Los ingenieros deben ser conscientes culturalmente, ágiles, flexibles y móviles (Continental, 2006). Están exigidos a responder a los cambios intelectuales, desde lo disciplinario hacia lo multidisciplinario, acomodando un enfoque mucho más holístico. (Sheppard, 2008).

La bibliografía estudiada reafirma el enfoque interdisciplinario que debe abordar la ingeniería, enfatizando especialmente la necesidad de integrar habilidades blandas a su formación académica.

En el área de economía y negocios, se destacan las observaciones realizadas en Carnegie Mellon University, en un programa ofrecido conjuntamente por profesores de la Escuela de Negocios Tepper y la Escuela de Diseño. Plantea que actualmente el enfoque del espíritu empresarial esta incluyendo la iniciativa de diseñar y liderar un negocio a través del trabajo en equipos Interdisciplinarios, ya que este enfoque combina las perspectivas necesarias para la comercialización eficaz de la innovación. Se declara además como factor esencial para el desarrollo de la interdisciplina, su incorporación en los programas de estudio, la capacidad de lograr un equipo docente interdisciplinario (interfacultades), la capacidad de colaborar y tener el respeto mutuo de lo que cada parte pone sobre la mesa. (Boni et al., 2012).

La bibliografía estudiada fundamenta el enfoque interdisciplinario que debe abordar economía y negocios, infiriendo por ejemplo, que las innovaciones potenciales no fracasan por falta de buenas ideas, sino por una falta de proceso que incorpore el aporte de las experticias de disciplinas distintas. Según Walter Lippman "Cuando todos los hombres piensan parecido; nadie piensa mucho".

Si contrastamos diseño, ingeniería, economía y negocios, cada disciplina tiene desafíos diferentes con los que proyecta mejorar su desempeño en el mundo actual, estos desafíos aunque distintos convergen en la necesidad de aprender a trabajar de manera conjunta (interdisciplinaria), en que hay que acercarse y valorar el aporte de otras disciplinas, $y$ en que no sólo las habilidades técnicas son importantes, sino además son altamente valoradas las habilidades blandas, entre ellas la creatividad para resolución de problemas.

Todas estas disciplinas, requieren evolucionar hacia los desafíos de hoy, acercarse hacia un trabajo interdisciplinario y creativo para dar respuesta a los problemas a los que se enfrenta la empresa.

\section{Desafíos interdisciplinarios en la formación universitaria}

El diseño responde a requerimientos interdisciplinarios, por esto la Facultad de Diseño de la Universidad del Desarrollo está trabajando para formar profesionales con visión global y competencias en trabajo colaborativo e interdisciplinario. La creatividad en el contexto de más de una disciplina es particularmente relevante en el campo del diseño.Y no sólo porque ésta es motor de la innovación, sino además porque el diseño per se es una disciplina que debe trabajar e interactuar no solo con, sino para otros.

La formación de estas competencias es un desafío impuesto por el mundo actual para todas las profesiones, atendiendo a ello la Universidad del Desarrollo desde el año 200I desarrolla con énfasis la temática del emprendimiento, identificándolo como uno de sus sellos fundacionales, lo que tiene positivos efectos curriculares y estructurales en los diferentes programas académicos de la Universidad. En este mismo proceso, se propuso institucionalmente desarrollar con fuerza la temática de la innovación, buscando complementar el espíritu y capacidad de emprendimiento de los egresados de la Universidad de Desarrollo con herramientas y métodos para la creación de valor en diversas áreas de su quehacer.

Es relevante orientar programas académicos hacia la formación de profesionales más innovadores para lograr una conexión entre las necesidades actuales y futuras de nuestra sociedad. Por una parte, la desafiante realidad de un país como Chile, ad portas del desarrollo, requiere esfuerzos institucionales por potenciar la capacidad de generación de valor por nuestra economía. Por otra parte, la apertura de nuestro mercado y cultura hacia otras fronteras, plantea el desafío de integrarnos, a la vez que competimos con nuevos actores. Por lo tanto, en busca de herramientas que permitan lograr lo anterior, las competencias que requieren los profesionales de los próximos 5 ó 10 años deben ser complementadas ya no sólo con su quehacer disciplinar, sino también con algún elemento diferenciador, el que identificamos y proponemos como innovación interdisciplinaria.

Referentes académicos de esta tendencia son el d.School de Stanford University y Olin College of Engineering en Estados Unidos; Rotman School of Management de Toronto University en Canadá y Aalto University en Finlandia. Todos ellos han desarrollado exitosos programas académicos que integran visiones multidisciplinarias, potenciando así las capacidades de sus estudiantes, tanto en pre como en postgrado.

El año 2009, las Facultades de Diseño, Ingeniería y Economía y Negocios, se reunieron a pensar cómo cruzar los caminos que cada una de ellas venía recorriendo -orientadas en un

ISSN: 07 I8-2724. (http://www.jotmi.org)

Journal of Technology Management \& Innovation (C) Universidad Alberto Hurtado, Facultad de Economía y Negocios. 
principio de forma individual- a la instalación de una cultura de innovación al interior de cada uno de sus programas curriculares. Con este propósito se crea iCubo, Instituto de Innovación Interdisciplinaria. La propuesta de valor de iCubo es la formación en innovación bajo una aproximación interdisciplinaria y su primer objetivo es la creación y promoción de innovación al interior de la Universidad en diversos ámbitos. Uno de ellos, es la dimensión curricular, para la que se desarrollan programas de iniciación en la innovación con cursos de amplia cobertura, un programa de entrenamiento de competencias de innovación en el pregrado llamado "dLab iCubo", y variados cursos orientados a educación de ejecutivos para la profesionalización y profundización de dichas herramientas. Asimismo iCubo desarrolla cursos, programas y proyectos para profesionales, emprendedores, empresas y organizaciones sociales.

El año 2010, la Universidad del Desarrollo firma un acuerdo con Stanford Technology Ventures Program (STVP), a través del Centro de Emprendimiento e Innovación de la Facultad de Ingeniería de Stanford University. Esta alianza permite la colaboración para el desarrollo de planes curriculares, de capacitación docente, y del desarrollo de la planta académica en función de la innovación en el contexto de iCubo,y con la misión de poder traspasar lo incorporado a nuevas y otras áreas de la universidad.

La concreción de iCubo marca un hito muy importante para la Facultad de Diseño manifiesto en los cimientos de dicha facultad desde su misión, la que señala que "La Facultad de Diseño de la Universidad del Desarrollo tiene por misión formar profesionales emprendedores, líderes e innovadores, capaces de integrar en el proceso proyectual, las distintas áreas del conocimiento y herramientas de la disciplina y que entiendan y valoren las metodologías de trabajo interdisciplinario como eje estructurante de su desempeño profesional, para contribuir al progreso económico, social y cultural del entorno en el que se desenvuelvan."

Actualmente, la Facultad de Diseño como parte integral de iCubo participa en su directorio, mesa ejecutiva y a través de la docencia con varios cursos para d-Lab y cursos de profundización para otras disciplinas dictados por profesores de la Facultad, entre ellos los cursos de Design Thinking, Prototipado Rápido y Creatividad para la Innovación.

El impacto de iCubo para la Universidad del Desarrollo apunta a posicionarla como un líder en la formación de innovadores y agentes de cambios, sea cual sea su destino profesional. Al mismo tiempo, este proyecto desarrolla la vinculación de la UDD con organizaciones productivas y sociales desde el punto de vista de generar oportunidades de solución a sus problemas. Además, iCubo potencia la interacción de la UDD con universidades extranjeras líderes y al mismo tiempo la posiciona como referente ante otras universidades de la región.

Este énfasis es consistente con la tendencia global que relacionaba la innovación con el trabajo multidisciplinario, con competencias profesionales claves para el siglo XXI, a través metodologías de trabajo aplicadas y enfoques tipo taller donde el aprendizaje se desarrolla de forma empírica, lo que clarificaba su relación con el emprendimiento, y que vincula en forma muy especial el mundo de los negocios, la ingeniería y el diseño.

Hoy, iCubo se encuentra en la etapa de implementación de su modelo académico concibiéndose desde la base como un centro para innovación que integra en la formación de sus alumnos, la ciencia y tecnología, la visión de negocios y el diseño.

Dado que la educación de diseñadores del siglo XXI requiere cada vez más formar emprendedores de sus propios proyectos, los diseñadores deberán ser capaces de integrar múltiples disciplinas para inventar el futuro. En este sentido, la formación que reciben los alumnos en el programa de pregrado que se da en iCubo permite una educación centrada justamente en estos aspectos. El diseño es entendido como parte de la solución.

Formar profesionales con capacidad de resiliencia, trabajo de colaboración con otros y co-creación, se enfoca en el nuevo paradigma de formación profesional que difiere de la mirada individualista más bien propia de la globalización (Manzini \& Vezoli, 2008). Así, la malla curricular de iCubo permite una mirada profunda en el quehacer práctico, en los equipos integrados, en las relaciones interpersonales y en la diversidad de las experiencias.

Específicamente, el programa de mayor complejidad desarrollado por este instituto es un programa que ha sido implementado a modo de piloto en el último año del pregrado de las tres facultades en cuestión. Se trata de una vía alternativa de cierre del ciclo de licenciatura para alumnos de las carreras de Diseño, Ingeniería Civil Industrial e Ingeniería Comercial, denominado dLab. Es un programa académico de un año de duración que sustituye el quinto año de estas tres carreras con un modelo de educación interdisciplinaria en el que los alumnos trabajan juntos desarrollando proyectos de innovación con organizaciones externas a la universidad, pudiendo de esta manera obtener resultados implementados $y$ generando valor real y demostrable al final de su ciclo académico. Durante toda la duración del proyecto son dirigidos por un tutor-profesor responsable de proceso, y apoyados por asesores-expertos en el área de trabajo en que desarrollan su proyecto, para finalmente generar un prototipo entregable. 
Curricularmente contempla dos líneas de cursos. Una línea teórica y otra práctica en paralelo. Los alumnos trabajan con la metodología del design thinking, propia de los procesos centrado en usuarios que buscan la colaboración radical en equipos multidisciplinarios para lograr la innovación (Brown, 2008). A través de esta metodología específicamente se busca entrenar en los alumnos la creatividad aplicada, para generar ideas originales, desarrollar nuevas aproximaciones a problemas, tomar riesgos y realizar contribuciones únicas, explorando las variables que estimulan o inhiben la creatividad y la innovación en individuos, equipos y organizaciones. Durante los distintos cursos se ejercitan procesos y herramientas de que permiten el desarrollo de la observación y registro para la empatía; la detección de oportunidades en forma temprana; y la capacidad de visualizar una idea a través de diferentes métodos de representación, que van desde lo conceptual, propio del mundo de la ideación, hasta lo concreto y material, en la creación y utilización de prototipos rápidos para la comunicación y evaluación de dichas ideas, entre otras competencias y herramientas.

A través de este programa se ha generado la posibilidad real de aplicar herramientas que fortalezcan y faciliten el trabajo en equipos interdisciplinarios, dotando de competencias y habilidades de innovación y emprendimiento a los alumnos que se preparan para el mundo profesional. Formando en ellos capacidad de generar impacto en la industria, el sector público y en todas aquellas instancias que requieran de una mirada distinta en pos del crecimiento, el desarrollo y el beneficio de las personas y el país. De manera tal que sea un aporte significativo en su proceso de integración al entorno laboral, generando valor y capital social ya sea como emprendedores o intraemprendedores.

\section{Bibliografía}

ALBORNOZ, C.A. (20II). Goals, Content, and Methods of Entrepreneurship Education: A Multiple Case Study. Florida International University.

BLAIR, B. (20I I). Elastic minds? Is the interdisciplinary/multidisciplinary curriculum equipping our students for the future: A case study. Art, Design \& Communication In Higher Education, I0(I), 33-50. doi:I0.1386/adch.10.1.33_I

BONI, Arthur A., Laurie R. Weingart, and Shelley Evenson. (2009) Innovation in an Academic Setting: Designing and Leading a Business Through Market-Focused, Interdisciplinary Teams. Academy Of Management Learning \& Education 8, no. 3 407-417. Business Source Complete, EBSCOhost (accessed November 10, 2012).

BROWN, Tim. (2008). Design Thinking. Harvard Business Review.

CHHABRA, Esha. (201I). Stanford's Institute of Design: School for world changers. Christian Science Monitor, September 30.N.PAG. Academic Search Complete, EBSCOhost (accessed November 10, 2012).

CINDA. (2000) Centro Interuniversitario De DesarrolloCinda. Fondo De Desarrollo Institucional. Ministerio De Educación-Chile. Las Nuevas Demandas Del Desempeño ProfesionalY Sus Implicancias Para La Docencia Universitaria.

DE GEUS,A.P. (1988). Planning as learning. Harvard Business Review, 88(2), 70-74.

DUDERSTADT, J. (2008). Engineering for a Changing World. A Roadmap to the Future of Engineering Practice, Research, and Education. The Millenium Project. The University of Michigan. United States.

DYM, C. L., Agogino, A. M., Eris, O., Frey, D. D., \& Leifer, L. J. (2005). Engineering Design Thinking, Teaching, and Learning. Journal Of Engineering Education, 94(I), 103-120.

HOLT, K. (1977). Creativity--a new challenge to the industrial engineer. International Journal of Production Research, I5(5), 4 II.

ITKONEN, M., Ekman, K., \& Kojo, I. (2009). Murjottelu - interdisciplinary training campaign for industrial design and engineering students. European Journal Of Engineering Education, 34(3), 263-27I. doi: 10.1080/03043790903038858

ISSN: 07 I8-2724. (http://www.jotmi.org) 
KAUFMAN, J. C., \& Beghetto, R. (2009). Beyond Big and Little:The Four C Model of Creativity. Review of General Psycology.

MAMYKINA, L., Candy, L., \& Edmonds, E. (2002). Collaborative Creativity. Communications Of The ACM, 45(I0), 96-99.

MANZINI, E., Vezzoli, C. (2008). Design for Environmental Sustainability. INDACO Department. Politécnico de Milán. Italia

MCDONALD, J. (20II). The Creative Spirit of Design. Techtrends: Linking Research \& Practice To Improve Learning, 55(5), 53-58. doi: | 0.1007/s | |528-0 | I-0528-2

OBRADORS, M. (2007). Creatividad y Generación de Ideas. Universidad Autónoma de Barceloma. Servei Publicaciones. Universidad de Valencia. España.

OECD. (2005) Selection and definition of Key competencies.

PARENTE, D. E. (2006) Advertising campaign strategy: A guide to market communication plans (4th ed.). Mason, $\mathrm{OH}$ : Thomson South Western.

PEREZ, C. (2002). Technological Revolutions and Financial Capital:The Dynamics of Bubbles and Golden Ages (p. 208). Edward Elgar Pub.

ROBINSON, K. (2006) The Element: How Finding Your Passion Changes Everything

SPOHRER, J., \& Maglio, P. P. (2008). The Emergence of Service Science: Toward Systematic Service Innovations to Accelerate Co-Creation of Value. Production and Operations Management, I7(3), 238-246. doi: I0.340 I/poms. I080.0027 